\title{
Impact of Shape and Total of Particle to Characteristics of Partial Discharge and Breakdown Voltage on Oil Insulation in Uniform Field
}

\author{
Daniar Fahmi ${ }^{1, *}$, I Made Yulistya Negara ${ }^{1}$, R. Wahyudi ${ }^{1}$, Dimas Anton Asfani ${ }^{1,2}$, Made Yudhi Setyawan ${ }^{1}$, and \\ Mochammad Wahyudi ${ }^{1}$ \\ ${ }^{1}$ Department of Electrical Engineering \\ Institut Teknologi Sepuluh Nopember (ITS) \\ Surabaya, Indonesia \\ ${ }^{2}$ Center of Excellence for Automotive Control \& System ITS \\ *daniarfahmi@ee.its.ac.id
}

\begin{abstract}
Oil insulation is one of the insulation medium types commonly used in high voltage apparatus. This insulation is to separate between the conductive part and non-conductive part. However, oil insulation can experience decreasing of dielectric strength. It may be caused by the presence of solid particles. Therefore, the testing was conducted to obtain the effect of solid particles to characteristics of partial discharge and breakdown voltage of oil insulation. The results generally proved that the existence of solid particles can reduce the breakdown voltage value of oil insulation. In addition, the solid particles led to two types of corona on oil insulation, namely positive and negative corona.
\end{abstract}

Keywords-oil insulation; partial discharge; breakdown voltage; corona; particle shape; particle total.

\section{INTRODUCTION}

Insulation medium is an isolator to separate two or more live electrodes. One of the insulation medium types is liquid insulation. Liquid insulation type has a greater dielectric strength compared to gas insulation type as liquid insulation has a density of 1000 times or more than gas insulation. Liquid insulation is commonly used as an insulation medium on capacitors and transformers. One of the liquid insulation types is oil.

Behind its excess as insulator, liquid insulation can also suffer breakdown voltage or insulation failure process. One of the causes of breakdown voltage is the existence of particle contaminants in oil insulation. In this case, dielectric strength of liquid insulation can be degraded by foreign particles, such as oxygen, water, sediment, and other particles as a result of solid decomposition [1].

Characteristic of breakdown voltage of oil insulation is significantly affected by the presence of solid particles. Therefore, this paper dealt with the effect of shape and total of solid particles to breakdown voltage on oil insulation.

\section{LIQUID INSULATION FAILURE DUE TO PARTICLE CONTAMINANT}

Solid particles on oil insulation may be sourced from the insulation degradation process, particles from outside that go inside during the operation, and contact to the outside environment on uncover apparatus. Those particles will lead to enlargement of field on the insulation material around the particles. Later, the field creates electric force that drives the particles toward greater field. The magnitude of electric force on solid particle is formulated as below:

$$
F=2 \pi r^{3} \varepsilon_{0} \varepsilon_{1} \frac{\varepsilon_{2}-\varepsilon_{1}}{\varepsilon_{2}+2 \varepsilon_{1}} \nabla E^{2}
$$

where: $F=$ force acting to particle, $r=$ radius of particle, $E=$ voltage gradient, $\varepsilon_{1}=$ liquid dielectric permittivity, and $\varepsilon_{2}=$ permittivity of particle.

If $\varepsilon_{2}>\varepsilon_{1}$, then the electric force working on particle was in the direction of the maximum electric pressure $\left(F_{a}\right)$ and pushes the particle toward the part that has a strong field. Conversely, if $\varepsilon_{2}<\varepsilon_{1}$, then the force is opposite to the maximum electric pressure $\left(F_{b}\right)$. When $\varepsilon_{2}>\varepsilon_{1}$, the dielectric constant of particle is larger compared to the dielectric around it. Consequently, when there is a high field in electrode, the particle will be drove toward the part with strong field. The particles that are attracted to a strong field will be charged due to the effect of field. Once the particle is charged, the particles will return to the electrode with weak field and will release the charge. This mechanism generates the micro discharge between particle and electrode [2].

In addition, there are forces of gravity and viscous affected to particles as illustrated in Fig. 1. The gravity force is the downward force acting on the particles, while the viscous force is the viscosity of insulation. Those forces will slow down the movement of particles. The magnitude of gravity and viscous forces is formulated as follow:

$$
F_{g}=\pi r^{2} \operatorname{l\rho g}
$$




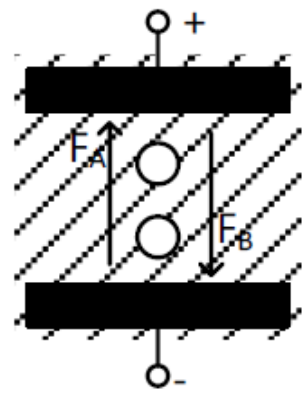

Fig. 1. The particle failure of oil insulation medium.

$$
F_{v}=6 \pi r v \eta
$$

where: $\rho=$ particle density $\left(\mathrm{kg} / \mathrm{m}^{3}\right), g=$ gravity acceleration $\left(9.8 \mathrm{~m} / \mathrm{s}^{2}\right), v=$ particle velocity, and $\eta=$ viscosity constant [3], [4].

All three forces work together on particles. If the electric field is greater than the forces of gravity and viscous, the particles will be pushed toward a strong field. When there are many particles in the dielectric, $F_{a}$ causes the particles to be attracted and parallel between the two electrodes as if to form a bridge that will initiate failure.

\section{EXPERIMENTAL SETUP}

This test used high DC voltage to investigate the impact of shape and total of particles to the characteristics of dielectric strength of oil insulation, namely partial discharge and breakdown voltage. The circuit of testing was shown in Fig. 2 and 3. The parts of the test circuit were as follows: $1=$ high voltage transformer, 2 = high voltage diode, $3=$ coupling capacitor, $4=$ measuring resistor, $5=$ plate electrode, $6=$ grounding switch, $7=$ current transducer, $8=\mathrm{PC}, 9=$ current probe.

The shape and total of solid particles were employed as variables. The particles were made from aluminum wire that was cut into small pieces with size variation of $2 \mathrm{~mm}, 4 \mathrm{~mm}$, and $6 \mathrm{~mm}$.

The test was performed based on the standard of breakdown voltage test, namely IEC 156 and SPLN 49-1 1982. The standards stated that the magnitude of breakdown voltage was $30 \mathrm{kV}$ and the distance of two electrodes was $2.5 \mathrm{~mm}$. However, this test used $10 \mathrm{~mm}$ of electrodes distance in order to obtain the effect of shape and total of particles to partial discharge and breakdown voltage of oil insulation. Furthermore, the chosen distance will easily facilitate to observe the symptoms of such phenomena during the test.

\section{RESULTS AND ANALYSIS}

\section{A. Particles Movement}

During the test, a video recording was conducted to investigate visually the movement of particles in oil insulation. The result of video recording was shown in the form of pictures as depicted on Fig. 4.

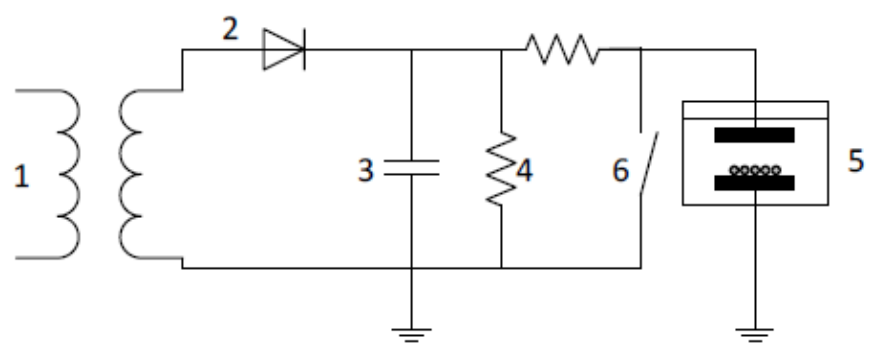

Fig. 2. The circuit of high dc test.

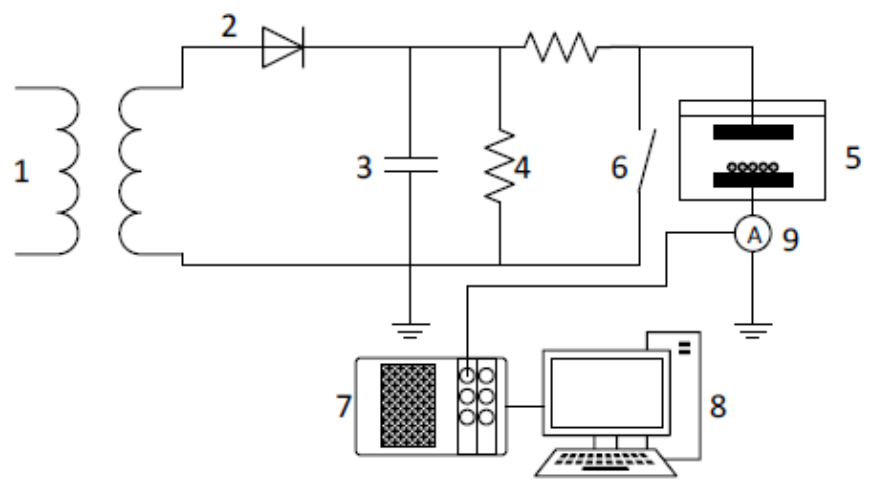

Fig. 3. The circuit of partial discharge test.

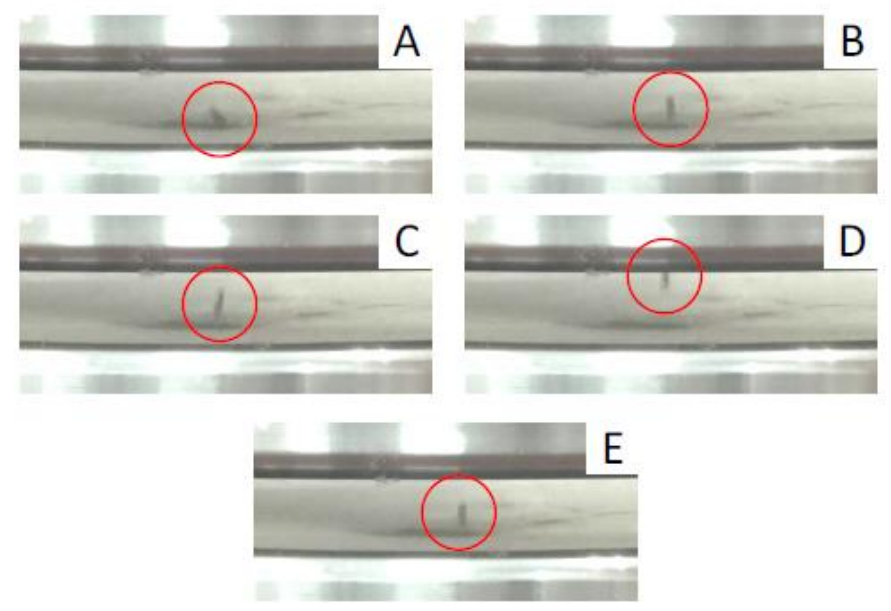

Fig. 4. The movement of particle in oil insulation.

\section{B. Partial Discharge and Breakdown Voltage}

The measurement of inception voltage was done to determine the effect of shape and total of particle to partial discharge and breakdown voltage in uniform field. In this case, the test was initially given to obtain the inception voltage value of partial discharge as seen in Table 1 and 2. The test was then continued by the breakdown voltage test as shown in Table 3.

\section{Mechanism of Particles Movement}

The movement of particles in oil insulation between two electrodes was the initial form of partial discharge. The particles movement was started by an increasing in voltage values on the electrodes and the forming of particles on the negatively charged electrode as depicted on Fig. 5. The establishment of particles was due to the presence of charge 
that began to be induced into the particles. The increasing of voltage on the electrode created the higher induced charged as equation below:

$$
Q=2 \pi \varepsilon_{0} r l E
$$

Equation (4) was the amount of charge that was induced when the particles were in horizontal position. On the other hands, the amount of charge that was induced when the particles were in vertical position was formulated as:

$$
Q=\frac{\pi \varepsilon_{0} l^{2} E}{\ln \left(\frac{2 l}{r}\right)-1}
$$

where: $E$ was field between electrodes, was permittivity of vacuum, was length of particle, and was radius of particle.

The difference in charge between particle and positively charged electrode resulted in a force that attracted the particles towards the electrode. In Fig. 6 (A and i), the particles have already been established. Due to the presence of a strong field on the positively charged electrode and the difference in charge between the positive electrode and the particle led to the particle to be pushed toward the positively charged electrode. This movement into a strong field was influenced by three forces acting on the particle, namely electric force, gravitational force, and viscous force. If the electric force was greater than the gravitational force and the viscosity force, so the particles would be pushed toward a strong field. The equations for the three forces were shown in (1) to (3).

Fig. 7 showed the particles that have been attached to the positively charged electrode. The strong field on the positively charged electrode was induced into particles, so that the particles would also be positively charged. The particles containing the positive charge would be pushed back to the negatively charged electrode due to the particles and electrodes having the same charge. The positively charge particles that was pushed into the negative electrode would release the charge upon arrival in the negative electrode, so that the micro discharge between the particles and the negatively charged electrode occurred.

TABLE I. INCEPTION VOLTAGE OF PARTIAL DiSCHARGE TO SHAPE AND LENGTH OF PARTICLE (WITH 0.5 MM RADIUS)

\begin{tabular}{cccccccc}
\hline \hline \multirow{2}{*}{1} & \multirow{2}{*}{ Total } & \multicolumn{7}{c}{ Voltage (kV) } \\
\cline { 2 - 8 } & & $I$ & $I I$ & $I I I$ & Average & Min & Max \\
\hline \multirow{2}{*}{2} & 1 & 20.5 & 19.8 & 20.3 & 20.20 & 19.8 & 20.5 \\
\cline { 2 - 8 } $\mathrm{mm}$ & 2 & 20.3 & 19.4 & 19.6 & 19.77 & 19.4 & 20.3 \\
\cline { 2 - 8 } & 3 & 20.1 & 19.8 & 19.2 & 19.70 & 19.2 & 20.1 \\
\hline \multirow{2}{*}{4} & 1 & 20.1 & 19.8 & 20.3 & 20.07 & 19.8 & 20.3 \\
\cline { 2 - 8 } $\mathrm{mm}$ & 2 & 19.8 & 19.4 & 19.7 & 19.63 & 19.4 & 19.8 \\
\cline { 2 - 8 } & 3 & 18.4 & 19.5 & 19.3 & 19.07 & 18.4 & 19.5 \\
\hline \multirow{2}{*}{6} & 1 & 19.1 & 18.6 & 18.5 & 18.73 & 18.5 & 19.1 \\
\cline { 2 - 8 } $\mathrm{mm}$ & 2 & 17.8 & 19.2 & 18.3 & 18.43 & 17.8 & 19.2 \\
\cline { 2 - 8 } & 3 & 17.3 & 16.6 & 17.2 & 17.03 & 16.6 & 17.3 \\
\hline \hline
\end{tabular}

TABLE II. INCEPTION VOLTAGE OF PARTIAL DISCHARGE TO RADIUS OF PARTICLE (WITH 4 MM LENGTH)

\begin{tabular}{cccccccc}
\hline \hline \multirow{2}{*}{$\begin{array}{c}\text { Radius } \\
(\mathbf{m m})\end{array}$} & Total & \multicolumn{7}{c}{ Voltage $(\mathbf{k V )}$} \\
\cline { 2 - 8 } & & $I$ & $I I$ & $I I I$ & Average & Min & Max \\
\hline \multirow{2}{*}{0.5} & 1 & 20.1 & 19.8 & 20.3 & 20.07 & 19.8 & 20.3 \\
\cline { 2 - 8 } & 2 & 19.8 & 19.4 & 19.7 & 19.63 & 19.4 & 19.8 \\
\hline \multirow{2}{*}{0.75} & 1 & 18.4 & 18.3 & 17.8 & 18.17 & 17.8 & 18.4 \\
\cline { 2 - 8 } & 2 & 17.7 & 17.5 & 18.2 & 17.80 & 17.5 & 18.2 \\
\hline \multirow{2}{*}{0.8} & 1 & 16.7 & 16.1 & 14.2 & 15.67 & 14.2 & 16.7 \\
\cline { 2 - 8 } & 2 & 14.2 & 13.8 & 14.6 & 14.20 & 13.8 & 14.6 \\
\hline \hline
\end{tabular}

TABLE III. BREAKDOWN VOLTAGE TO PARTICLE SizE

\begin{tabular}{cccc}
\hline \hline Radius (mm) & Length $(\mathbf{m m})$ & Total & Voltage $(\mathbf{k V})$ \\
\hline \multirow{2}{*}{0.5} & 2 & 3 & 101 \\
\cline { 2 - 4 } & 4 & 3 & 96 \\
\cline { 2 - 4 } & 6 & 3 & 93 \\
\hline 0.75 & 4 & 3 & 90 \\
\hline 0.8 & 4 & 3 & 86 \\
\hline \hline
\end{tabular}
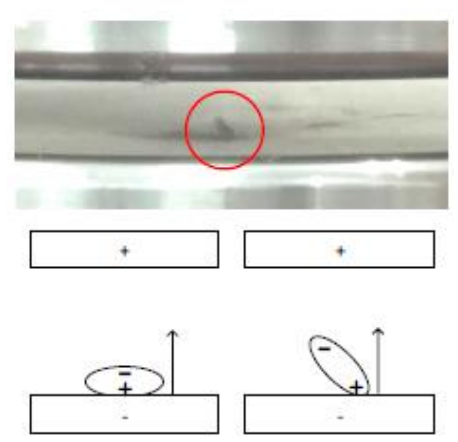

Fig. 5. Initial movement of particle.

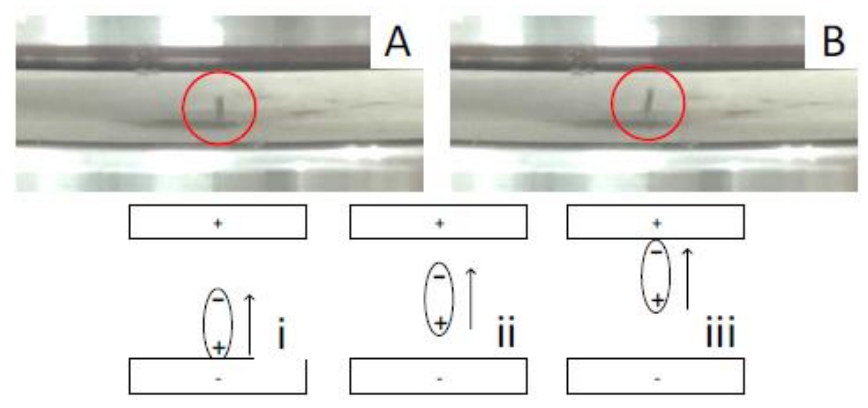

Fig. 6. The particles moving to positive electrode.
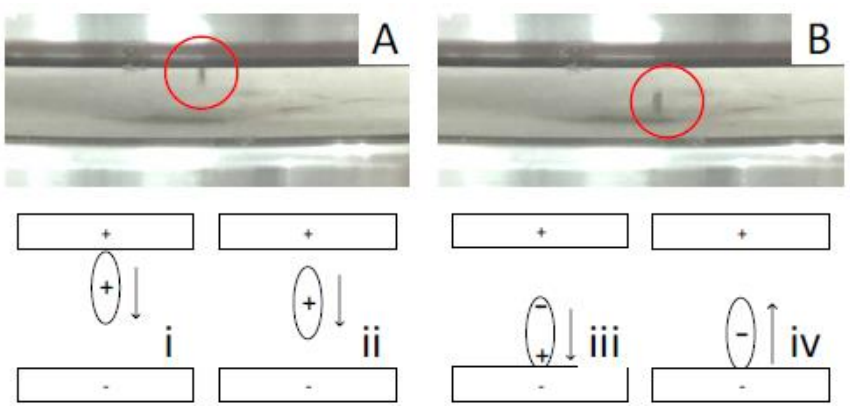

Fig. 7. The positively charged particles. 
The micro discharge was the release of charge between particles and electrodes in small quantity. The release of the charge on the particles would cause the particles to be negatively charged and would be drawn back to the positively charged electrode. The phenomena as shown in Fig. 5 to 7 would occur continuously, so that the particles move bouncing between two electrodes.

\section{Effect of Particle to Partial Discharge Inception Voltage}

The graph that showed the characteristic of partial discharge inception voltage as a function of difference of size and total of particles was depicted based on the Table 1. Fig. 8 revealed the effect of particle sizes to the partial discharge inception voltage in which the longer particle size so the smaller inception voltage of partial discharge. It may be caused by the effect of field around the particles. As stated in (4), the charge in particles was proportional to the length of particles. The longer particles so the greater charge contained in particles. Increasing of particles total would increase the field formed. The presence of an increasingly large field would cause the lower initial voltage.

The same condition was seen in Fig. 9. This figure showed the characteristic of initial voltage to the total of particle. It was seen that the total of particle was inversely proportional to the inception voltage of partial discharge.

The characteristic of partial discharge inception voltage to the particle radius was depicted on Fig. 10. The higher particle radius so the lower partial discharge inception voltage. It was due to the uniformity of field in the end of particle. The wider particle section the more uniform field. This would result in reducing of partial discharge inception voltage.

\section{E. Effect of Particle to Breakdown Voltage}

The result of breakdown voltage test was shown in Table 3. The measured breakdown voltage was inversely proportional to the particle length as the longer particle so the more charge contained in particle. This relation had been formulated in (1). The breakdown voltage occurred when the particles were floated between the two electrodes. When there was enough field and charge in particles, the breakdown voltage would appear between the electrodes.

Another result that could be seen in Table 3 was the breakdown voltage was inversely proportional to the radius of particle. The larger cross section of particle created the greater charge of particle. If there was enough charge in particle and strong field in electrodes, then there would be the phenomenon of breakdown voltage.

\section{F. Corona Mechanism}

The moving particle that bounced between the two electrodes resulted in the corona on the two ends of particle. The mechanism of the occurrence of corona due to the movement of particle was depicted on Fig. 11. There were two corona types in particle, namely positive corona (KP) and negative corona $(\mathrm{KN})$. The mechanism of corona event was explained in the following steps (i - vi).

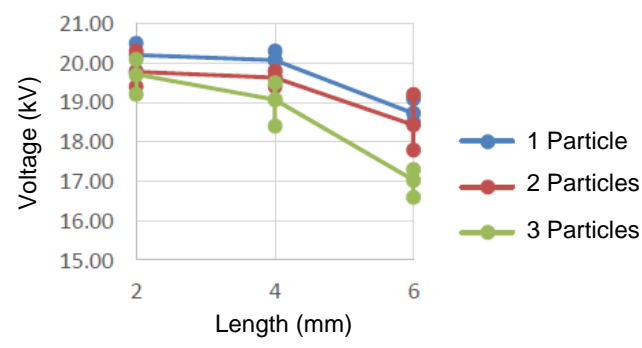

Fig. 8. The graph of inception voltage to particle size (with $0.5 \mathrm{~mm}$ radius).

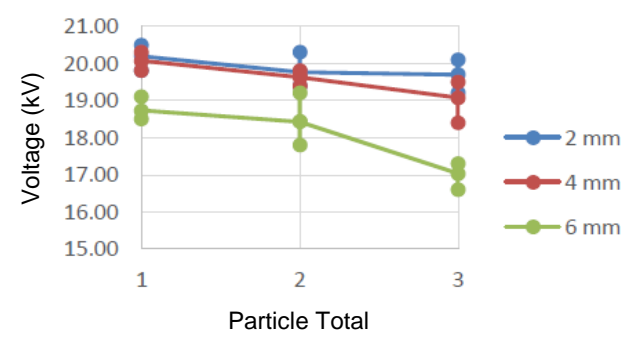

Fig. 9. The graph of inception voltage to particle total (with $0.5 \mathrm{~mm}$ radius).

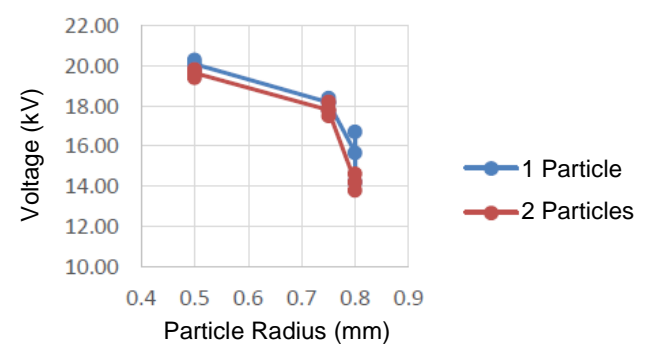

Fig. 10. The graph of inception voltage to particle radius (with $4 \mathrm{~mm}$ length).

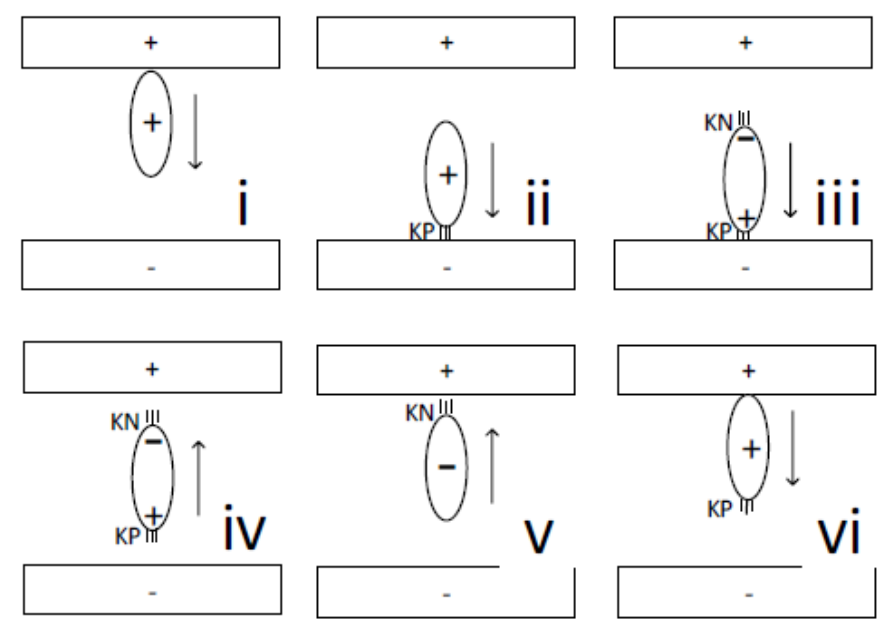

Fig. 11. The corona mechanism. 
i. The particles were pushed toward the negative electrode due to the equality of charge between the particles and the positive electrode.

ii. The particles currently on the negative electrode would release the charge (discharge) on the negative electrode, so that the positive corona appeared between the positively charged particle and the negative electrode.

iii. The release of positive charge on particles caused the top part of particle to be negatively charged and it may result in negative corona between the particles and the positive electrode at the strong field.

iv. The difference between the particle and the positive electrode caused the particle to be attracted toward the positive electrode. At the strong field, the movement of particles would generate the bicorona that was the occurrence of two corona type at the end of particle.

v. The particles that were negatively charged on the positive electrode provided the positive corona.

vi. The particle would contain the positive charge due to the strong field on the positive electrode. The positively charged particle triggered the positive corona between the particle and the negative corona.

\section{G. Corona Characteristics}

The current of corona at $20 \mathrm{kV}$ was depicted on Fig. 12(a). The corona began with the instability of distance between one current signal and another. As the voltage of electrode continuing to increase resulted in the higher corona current with high quantity. In addition, the distance between one current signal and the other current signals was stabilized. The increase of current value was due to the greater voltage value.

The characteristic of corona current at $80 \mathrm{kV}$ was shown in Fig. 12(c). The corona current was seemed to be greater and in higher quantity. Besides, the higher particle total so the greater current. It was caused by the rapid movement of particle between the two electrodes.

As seen in Fig. 12(c), the corona current could not be separated between the positive corona and the negative corona. This was due to the rapid movement of particle and the amount of corona current that occurred in large quantity. The high corona current value in Fig. 12(c) was the corona current when bicorona occurred on the particle. It was because the value of bicorona current was a combination of positive corona and negative corona.

If the shape of graph of corona current was enlarged, so it would look like the graph in Fig. 13. There were parts of $A$ and $B$ in the figure. The $A$ part was the current triggering the corona, while the $B$ part was the corona current in which the part consisted on negative and positive ions.

\section{H. Effect of Shape and Total of Particle to Corona Current Characteristic}

The test results were shown in Fig. 14 and 15. From the figures, the current did not have stability of distance between one current and other currents. The current forms at the particle size variation of $2 \mathrm{~mm}, 4 \mathrm{~mm}$, and $6 \mathrm{~mm}$ were difficult to be determined its characteristic. This was because the pattern of the current graphs that could not be determined. In addition, it was not easy to separate between positive corona current and negative corona current.
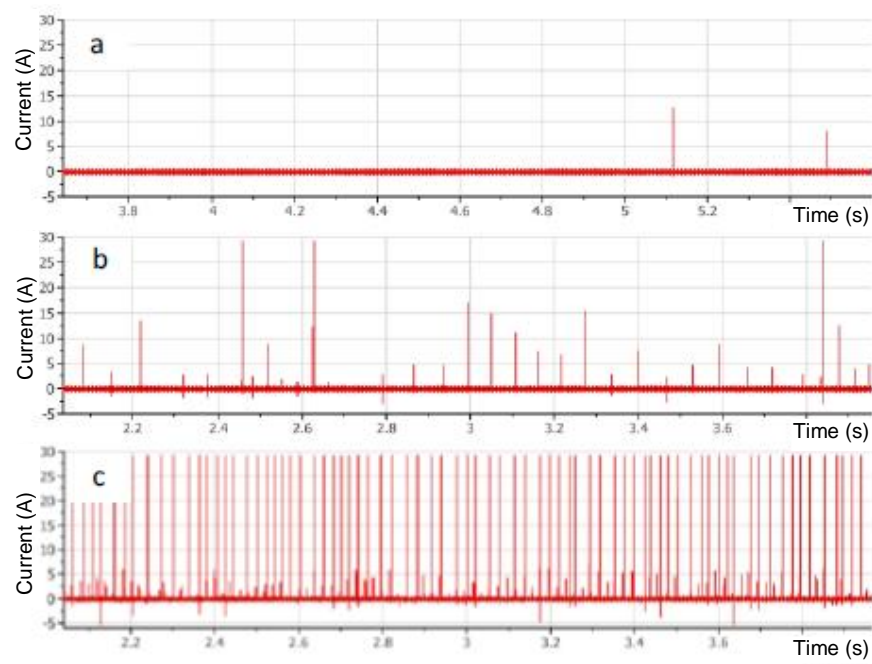

Fig. 12. The corona current at 1 particle with $0.5 \mathrm{~mm}$ radius and $2 \mathrm{~mm}$ length: a) $20 \mathrm{kV}$, b) $40 \mathrm{kV}$, c) $80 \mathrm{kV}$.

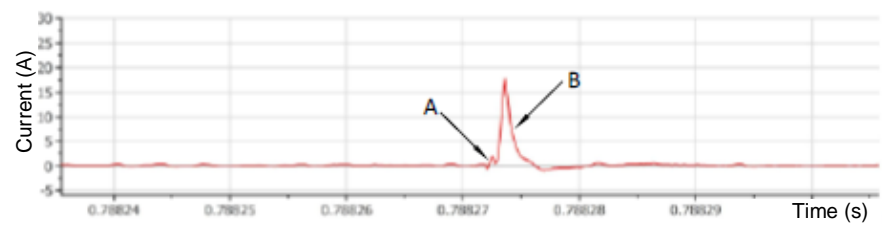

Fig. 13. The enlarged corona current.
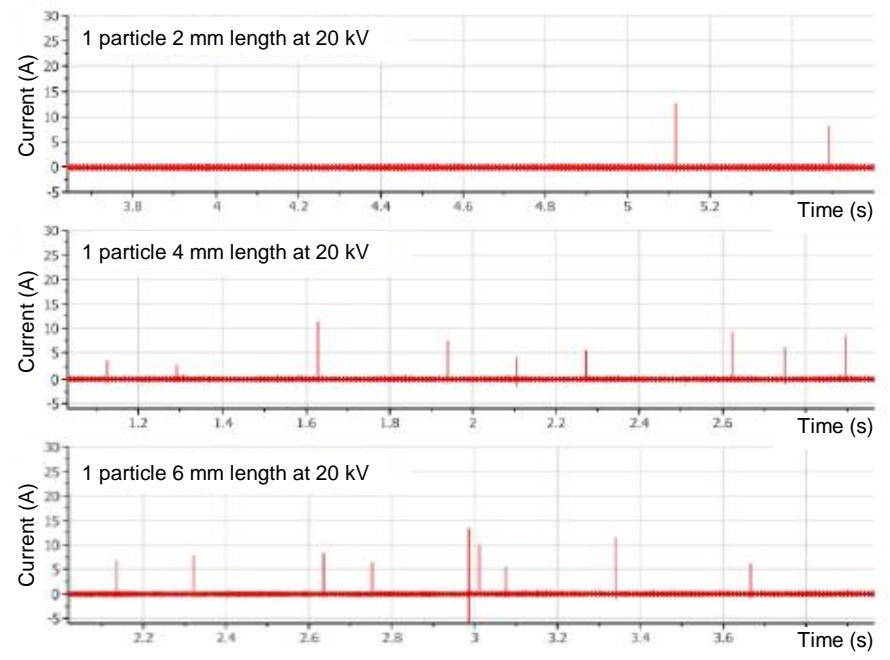

Fig. 14. The effect of particle size to corona current at $20 \mathrm{kV}$ (with $0.5 \mathrm{~mm}$ radius).

The current values in Fig. 15 were assumed that a current value smaller than $20 \mathrm{~A}$ was called as a minor pulse, whereas a larger value was called as a major pulse. Moreover, the average 
value of minor current on particle with $6 \mathrm{~mm}$ length was higher compared with other variations. The charge stored in particle with $6 \mathrm{~mm}$ length was greater than others may explain that result. Consequently, it created in higher partial discharge.

The similar result was seen in Fig. 16. It was difficult to determine the difference and pattern between the graphs. No positive and negative corona current could be separated. In addition, if the value of minor and major pulses was analyzed, then there was a difference between the two images. The minor pulse value of particle with $0.75 \mathrm{~mm}$ radius was lower than the particle with $0.55 \mathrm{~mm}$ radius. It was also caused by the amount of charge contained in the particles. The larger radius, the more charge contained in the particle. Consequently, the average current value during partial discharge was also greater. However, when the radius was increased to $0.8 \mathrm{~mm}$, the minor pulse was very small and in little quantity. It was because the field on the particle increasingly uniform, so that the generating of corona was difficult.
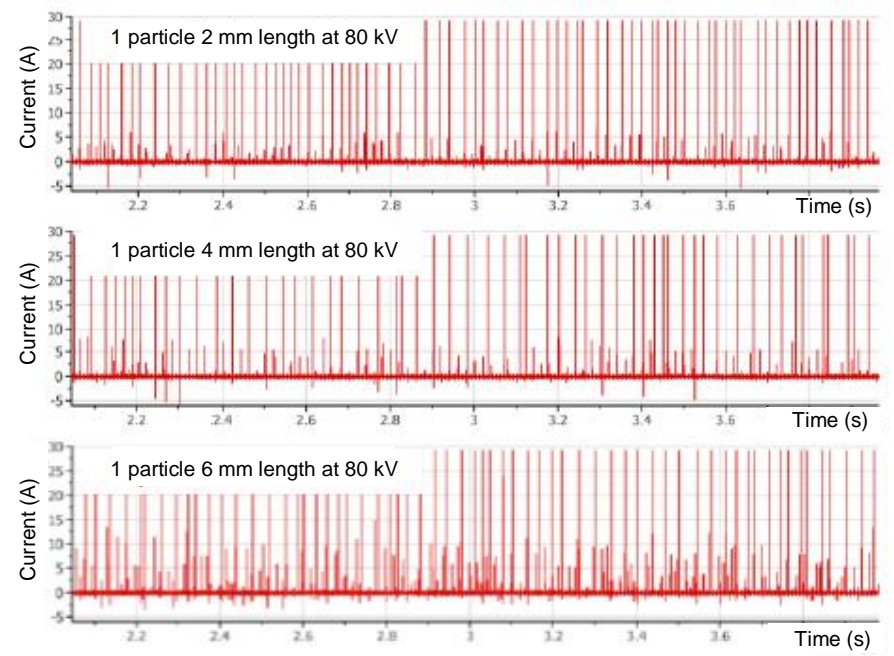

Fig. 15. The effect of particle size to corona current at $80 \mathrm{kV}$ (with $0.5 \mathrm{~mm}$ radius).
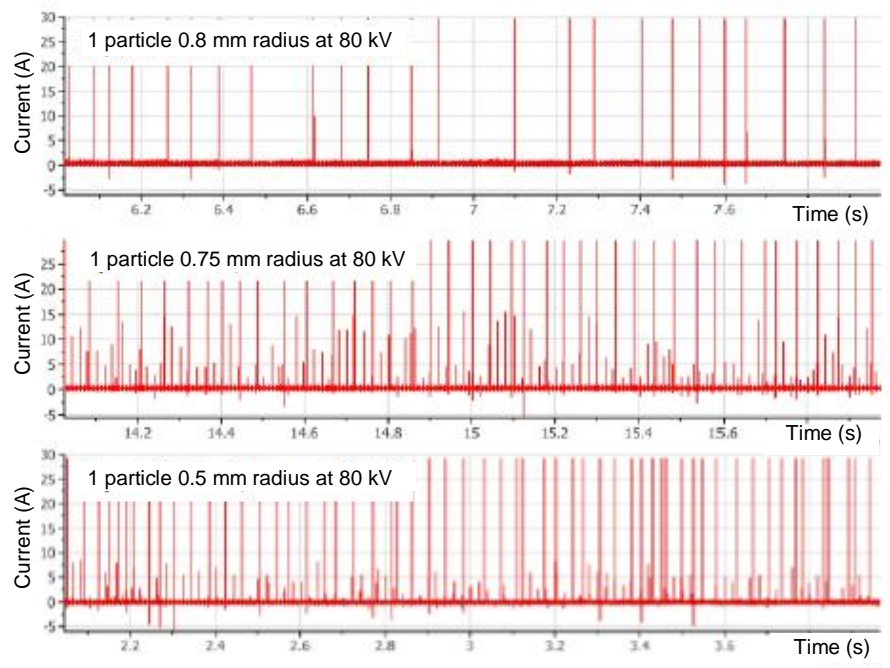

Fig. 16. The effect of particle radius to corona current at $80 \mathrm{kV}$ (with $4 \mathrm{~mm}$ length).

\section{CONCLUSION}

The size of particle was inversely proportional to the partial discharge inception voltage. The initial voltages of particle with length variation of $2 \mathrm{~mm}, 4 \mathrm{~mm}$, and $6 \mathrm{~mm}$ were 20.20 $\mathrm{kV}, 20.07 \mathrm{kV}$, and $18.03 \mathrm{kV}$, respectively. In addition, the total of particle was reversely proportional to partial discharge inception voltage. There were three types of particle total, namely 1,2 , and 3 . In 1 , the particle with $2 \mathrm{~mm}$ length had the inception voltage of $20.20 \mathrm{kV}$. In 2 , the particle had $19.77 \mathrm{kV}$, while in 3 the particle had $19.7 \mathrm{kV}$. The length and radius of particle were also inversely proportional to the breakdown voltage. The breakdown voltages for particles with $2 \mathrm{~mm}, 4$ $\mathrm{mm}$, and $6 \mathrm{~mm}$ (with $0.5 \mathrm{~mm}$ radius) were $101 \mathrm{kV}, 96 \mathrm{kV}$, and $93 \mathrm{kV}$, respectively. On the other hands, the breakdown voltages for particles with radius of $0.5 \mathrm{~mm}, 0.75 \mathrm{~mm}$, and 0.8 mm were $96 \mathrm{kV}, 90 \mathrm{kV}$, and $86 \mathrm{kV}$.

The presence of particles led to the two type of corona, namely positive and negative corona. The determining of corona characteristic was difficult since the pattern of graphs itself was not distinguished clearly. Moreover, the amplitude and corona current was uncertain. There was a different in minor pulse in the effect of corona to particle length. The average value of minor pulse was proportional to the length particle.

\section{REFERENCES}

[1] I.M.Y. Negara, Technique of High Voltage: Principle and Pratical Application (in Indonesian), Yogyakarta: Graha Ilmu, 2013.

[2] E. Koffel, W.S. Zaengl, and J. Kuffel, High Voltage Engineering Fundamentals, Oxford: Butterworth-Heinemannn, 2000.

[3] M. Holmberg, Motion of Metallic Particles in Gas Insulated Systems. Goteborg: Chalmers University of Technology, 1997.

[4] Y. Khan, Particle Triggered Breakdown Characteristics and Methods of Particle Deactivation around Spacers in Simulated GIS. Fukuoka: Kyushu University, 2004. 